
\title{
25 Research Soure \\ Evaluating the Covalent Binding of Carbapenems on BlaC Using Noncovalent Interactions
}

\section{Lu Sun}

Dalian University of Technology

\section{Hongjun Fan}

Dalian Institute of Chemical Physics

Shijun Zhong ( $\nabla$ sjzhong@dlut.edu.cn )

Dalian University of Technology School of Bioengineering https://orcid.org/0000-0002-4213-2808

\section{Research Article}

Keywords: Class A $\beta$-lactamase (BlaC), covalent carbapenem inhibitor, molecular dynamics simulation, binding free energy, consensus score

Posted Date: February 9th, 2021

DOI: https://doi.org/10.21203/rs.3.rs-178377/v1

License: @ (i) This work is licensed under a Creative Commons Attribution 4.0 International License. Read Full License 


\title{
Evaluating the Covalent Binding of Carbapenems on BlaC Using Noncovalent Interactions
}

\author{
Lu Sun ${ }^{1}$, Hongjun Fan², Shijun Zhong 1,* \\ ${ }^{1}$ School of Bioengineering, Dalian University of Technology, Dalian, Liaoning, 116024, China \\ ${ }^{2}$ State Key Laboratory of Molecular Reaction Dynamics, Dalian Institute of Chemical Physics, Chinese \\ Academy of Sciences, Dalian, Liaoning, 116023, China
}

Received: Day Month Year / Accepted: 28 Jan 2021

\begin{abstract}
Carbapenems, as irreversible covalent binders and slow substrates to the Class A $\beta$ lactamase $(\mathrm{BlaC})$ of Mycobacterium tuberculosis, can inhibit $\mathrm{BlaC}$ to hydrolyze the $\beta$-lactam drugs which are used to control tuberculosis. Their binding on $\mathrm{BlaC}$ involves covalent bonding and noncovalent interaction. We introduce a hypothesis that the noncovalent interactions dominate the difference of binding free energies for covalent ligands based on the assumption that their covalent bonding energies are same. MM/GBSA binding free energies calculated from the noncovalent interactions, provided a threshold with respect to the experimental kinetic data, to select slow carbapenem substrates which were either constructed using the structural units of experimentally identified carbapenems or obtained from the similarity search over the ZINC15 database. Combining molecular docking with consensus scoring and molecular dynamics simulation with MM/GBSA binding free energy calculations, a computational protocol was developed from which several new tight-binding carbapenems were theoretically identified.
\end{abstract}

Keywords Class A $\beta$-lactamase $(\mathrm{BlaC}) \cdot$ covalent carbapenem inhibitor $\cdot$ molecular dynamics simulation $\cdot$ binding free energy $\cdot$ consensus score

\section{Introduction}

Mycobacterium tuberculosis, a pathogenic bacterial species in the family of Mycobacteriaceae, causes tuberculosis which is one of the top 10 causes of death and makes millions of people fall sick each year.[1] This is complicated by the emergence of multidrug-resistant and extensive drug-resistant strains of Mycobacterium tuberculosis, which reveals the drawback of the current treatment strategies for tuberculosis. [1-3] The $\beta$-lactam class of antibiotics such as penicillin were effective in the treatment of tuberculosis due to their inhibition of bacterial cell wall synthesis.[4] However, the efficacy is reduced due to the resistance caused by Class $\mathrm{A} \beta$-lactamase (BlaC) which inactivates the $\beta$-lactam antibiotics via serine acylation followed by hydrolytic deacylation.[5] This involves the nucleophilic attack of the serine residue against the $\beta$-lactam carbonyl to form a covalent acyl-enzyme complex while the $\beta$-lactam ring is opened,[6] followed by the hydrolysis of the ester bond to release the inactive ring-opened product.[7] In order to

\footnotetext{
*Shijun Zhong: sjzhong@dlut.edu.cn
} 
prevent this mechanism of resistance, it is necessary to find novel compounds to inhibit $\mathrm{BlaC}$ hereby being of utility in $\beta$-lactam/ $\beta$-lactamase inhibitor combinations such as meropenem/vaborbactam[8] and meropenem/clavulanate.[9]

Carbapenems also contain the $\beta$-lactam ring and can be used as antibiotics targeting penicillin-binding proteins.[10] They are poor substrates with slow hydrolysis on BlaC and could act as inhibitor of BlaC or an active partner with inhibitor clavulanate[9] such that they may act as a template for the development of novel inhibitor combinations. The crystal structures of BlaC-carbapenem complexes have been reported for some carbapenems including meropenem (MERO),[9] doripenem (DORI) and ertapenem (ERTA) (Fig. 1a),[11] which provide structural basis for the design of new carbapenems using computational approaches. Kinetic parameters $k_{\text {cat }}$ and $K_{\mathrm{m}}$ have been measured for some carbapenems including the above three carbapenems, which are poor substrates with small $k_{\text {cat }}$ and $K_{\mathrm{m} .[12]}$ The structures of these carbapenems contain two parts, as shown in Fig. $1 \mathrm{~b}$, the core structure in blue and the $\mathrm{R}$ group in black. All carbapenems have the same core structure but different $\mathrm{R}$ groups.

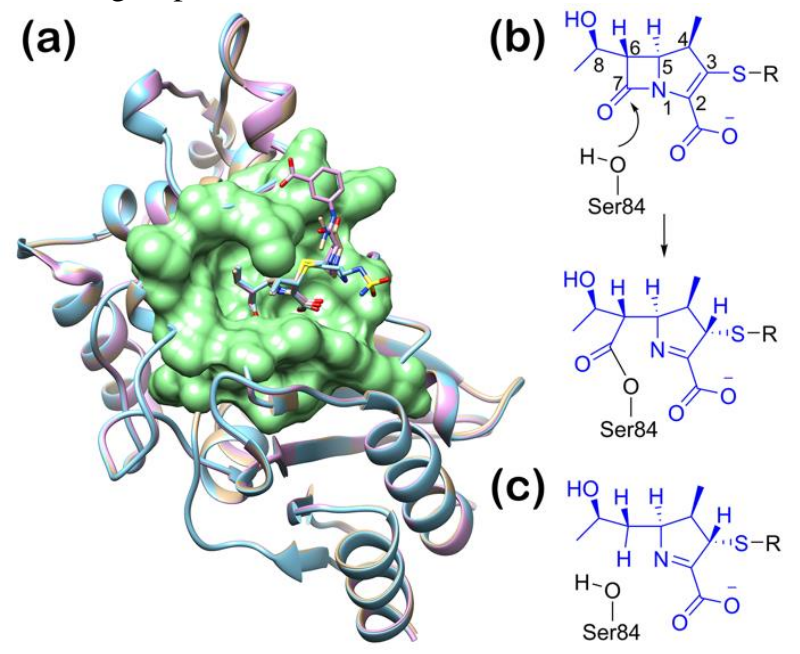

Fig. 1. (a) Superimposition of the three BlaC-carbapenem crystal structures. BlaC is shown in cartoon while the carbapenems are shown as sticks (DORI carbons in sky blue, ERTA carbons in plum and MERO carbons in tan). The binding site is expressed in surface using the structure of BlaC-MERO (PDB ID: 3DWZ). (b) Structural change during the covalent binding, i.e. the opening of $\beta$-lactam ring and the isomerization of pyrroline ring. (c) Structure used in molecular dockings.

The core structure of the carbapenems contains two rings, the $\beta$-lactam ring with the substituent $6-\alpha-1 \mathrm{R}$-hydroxyethyl and the pyrroline ring to which the $\mathrm{R}$ group is attached via the thioether sulfur atom on $\mathrm{C} 3$ atom. There are four chiral centers in the core structure, i.e. the carbon atom $\mathrm{C} 4$ (the stereoisomer $\mathrm{R}$ ) in the pyrroline ring, $\mathrm{C} 5(\mathrm{~S})$ at the joint of the pyrroline ring and the $\beta$-lactam ring, $\mathrm{C} 6(\mathrm{~S})$ in the $\beta$-lactam ring and $\mathrm{C} 8(\mathrm{R})$ in the substituent 6- $\alpha$-1R-hydroxyethyl group (Fig. 1b). The core part completely lies in the 
binding site while the $\mathrm{R}$ group spreads out of the binding site and is flexible (Fig. 1a). The $\beta$-lactam ring sterically blocks the binding site of $\mathrm{BlaC}$ and prevents its attack on the $\beta$ lactam class of antibiotics.[9,11] The binding contains both covalent and noncovalent interactions. Here, it should be pointed out that Ser84 is numbered according to the crystal structures, $[9,11]$ while Ser70 was used to indicate the same residue in the literature according to the Ambler position.[6,7,13] The noncovalent interactions involve the residues Ile117, Ser142, Glu182, Thr232, and Thr253 of BlaC.

The $\mathrm{R}$ groups cause the differences between these carbapenem molecules since they have the same core structure. Some carbapenems with different $\mathrm{R}$ groups were experimentally evaluated,[12] which may be used to build models to develop more efficient inhibitors based on variations of the $\mathrm{R}$ group. There can be many ways to construct the $\mathrm{R}$ group. In general, arbitrary permutations and combinations of any chemical structural units can generate a large number of structures. However, it might be restricted by chemical synthesis. In the present paper, we show how to build new R groups using the structural units of these experimentally identified carbapenems. Meanwhile, similarity search is used to find carbapenem-like structures. All these structures are then compared via analyzing the BlaC-carbapenem intermolecular interactions using computational tools with our filtering and scoring treatments.

Several computational methods already address covalent binding. CovalentDock[14] forms the covalent bond via a dummy atom, then computes the covalent bonding using a Morse potential which was fitted to the enthalpy change on bond length based on quantum chemical calculations. CovalentDock uses the AutoDock scoring function for the noncovalent interaction. Another covalent docking tool CovDock[15] forms the covalent bond using the OPLS3 force field for initial ligand binding poses generated by Glide docking sampling, then optimizes the linked structures using Prime, and ranks the binding poses using VSGB2.0 scoring function which is based on OPLS force field and Generalized Born solvation model. The covalent binding affinity can be computed in two individual states separately, i.e. the covalent binding and noncovalent binding states, using the free energy perturbation coupled with $\lambda$-exchange molecular dynamics method, while a $\mathrm{QM} / \mathrm{MM}$ calculation of warhead was also performed to address the noncovalent interactions. The calculations of a series of $\alpha$-ketoamide analogues relative to a common warhead scaffold revealed that covalent and noncovalent binding states of an inhibitor do not necessarily exhibit the same selectivity.[16]

In the present work, we show how to use the noncovalent interactions to evaluate the covalent ligands and help design new covalent carbapenems. First, MD simulations were applied to the BlaC-carbapenem covalent systems to evaluate the role of $\mathrm{R}$ groups by analyzing the structural difference and energy contributions. Then, some new compounds were built using the structural units in the R groups. Meanwhile, a similarity search with respect to the core structure of carbapenem, performed over ZINC15 database,[17] provided more compounds which were screened using molecular dockings. Furthermore, all selected compounds were consequently evaluated using MD simulations and the 
MM/GBSA binding free energy calculations. Finally, some compounds will be suggested for further experimental validation.

\section{Methods and Computational Details}

\subsection{Structure preparation}

The crystal structures of BlaC-carbapenem complexes were obtained from the Protein Data Bank (http://www.rcsb.org/),[18] including the complexes with DORI (PDB ID: 3IQA),[11] ERTA (PDB ID: 3M6H)[11] and MERO (PDB ID: 3DWZ),[9] respectively. The root-mean-square deviations (RMSDs) between any pair of the three structures are very small $(0.10 \sim 0.15 \AA)$ with respect to either the $\mathrm{C} \alpha$ atoms of the whole backbone or the atoms of the binding site residues within $6 \AA$ around the ligand (Table S1). Therefore, any of them, here 3DWZ, can be used as a representative to compute the binding of carbapenem ligands. The binding of carbapenem to $\mathrm{BlaC}$ causes a structural change which involves the formation of a covalent bond with the hydroxyl oxygen of Ser84, the opening of the $\beta$ lactam ring, and the isomerization of the pyrroline ring (Fig. 1b). The resulting structure is the conformation of carbapenem in the crystal and was used in our MD simulations.

The protonation of the protein was prepared using Tleap module in AMBER 14.[19] The histidine tautomers were determined using our in-house program which use criteria similar to those used in literature such as that by Baker and Hubbard.[20] We use two criteria to judge if a histidine $\mathrm{N}$ atom will be protonated. The first criterion is the possibility to form a hydrogen bond (H-bond) with an H-bond acceptor at a proper position. The second criterion is if there is a spacious cavity to avoid repulsion with other possible hydrogen atom on an $\mathrm{H}$-bond donor such as the amide nitrogen. A complementary convention is that the delta position is usually protonated if both $\mathrm{N}$ positions have no chance to form $\mathrm{H}$-bond and the cavities are spacious. It should be very strict to assign the protonation to both $\mathrm{N}$ atoms unless they can form very strong $\mathrm{H}$-bond with the surrounding $\mathrm{H}$-bond acceptors or if the residue is adjacent to a negative, acidic group.

\subsection{Parametrization}

Parametrization for the covalently bonded residue-ligand structure was performed using tools implemented in the AMBER package. There is a covalent bond between the residue Ser84 and ligand carbapenem. Therefore, this residue should be a new type, named SEP herein. The new residue SEP does not have the hydroxyl hydrogen atom on its sidechain, but leaves a linking site for forming a bond with ligand carbapenem. The partial atomic charges for SEP and ligand carbapenem were derived from the RESP (restrained electrostatic potential) charge model using the antechamber module implemented in AMBER, on the basis of the molecular electrostatic potential (ESP) calculated at HF/6$31 \mathrm{G}^{*}$ level using Gaussian 09[21] for a molecular model which contains the ligand 
carbapenem and the covalently bonded serine. Here, this serine is capped with $\mathrm{CH}_{3} \mathrm{CO}$ - at its $\mathrm{N}$ terminal and with $-\mathrm{NHCH}_{3}$ at its $\mathrm{C}$ terminal. The above ESP computation and RESP charge fitting were repeated for each individual SEP-ligand system. More details of the parameterization for these carbapenems and the covalently bonded serine SEP are given in Table S2.

\subsection{MD simulation and binding free energy calculations}

The protein-ligand systems were submitted to MD simulations using AMBER 14 package[19] with the AMBER ff14SB force field for protein[22] and the General Amber Force Field for ligand.[23] The process of MD simulation is described in Table S3. The MD trajectories were analyzed using CPPTRAJ module[24] implemented in AMBER 14 to derive the information about RMSD, root-mean-square fluctuation (RMSF) and Hbonds. The conformations in the trajectories were extracted for clustering using NMRCLUST[25] to obtain representative structures for further analysis of the binding modes in the active site. The details of clustering are given in Table S4.

Conventional binding free energy calculations via MD simulations cannot be directly applied to the covalently-bonded system. Fortunately, all carbapenems have same covalent bonding. Thus, the comparison between different carbapenems can be estimated by considering the noncovalent interactions. Therefore, we introduced a hypothesis that the difference of binding free energy for different carbapenems is determined by the noncovalent interactions if the covalent bonding energies are same to all carbapenems. The binding free energy $\Delta G_{b i n d}$ can be expressed as the following equation.

$$
\Delta G_{\text {bind }}=\Delta G_{\text {covalent }}+\Delta G_{\text {noncovalent }} \approx \text { constant }+\Delta G_{\text {noncovalent }}
$$

In the AMBER MM/GBSA binding free energy calculation, the receptor and ligand can be normally specified, no matter of covalent bonding or noncovalent interaction. By this way, the binding free energy for noncovalent interactions is a relative value which is calculated in AMBER as follows:

$$
\Delta G_{\text {bind }}=\Delta E_{\text {intermal }}+\Delta E_{v d w}+\Delta E_{\text {ele }}+\Delta G_{G B}+\Delta G_{S A}
$$

where $\Delta E_{\text {intermal }}$ is the change of the internal energy including the contributions from bonds, angles, and dihedrals, $\Delta E_{v d w}$ the non-bonded van der Waals interaction and $\Delta E_{\text {ele }}$ the electrostatic interaction. $\Delta G_{G B}$ and $\Delta G_{S A}$ represents the polar and nonpolar solvation energy, respectively, where GB stands for the Generalized Born model and SA for the solvent accessible surface area according to the MM/GBSA model[26] implemented in AMBER 14 suit,[19] using the model option GB2.[27,28] 


\subsection{Similarity search and molecular docking}

For finding more similar compounds, a similarity search, with respect to the ring part of the core structure of carbapenem (SMILES: $\mathrm{O}=\mathrm{C} 1 \mathrm{CC} 2 \mathrm{CC}=\mathrm{CN} 12$, InChl Key in the ZINC scaffold set: YZBQHRLRFGPBSL-UHFFFAOYSA-N ), was performed online (http://zinc15.docking.org/) using the default similarity criteria over the ZINC15 database which contains more than 750 million purchasable compounds.[17] A total of 243 similar molecules were obtained and further screened via a preliminary visual inspection to check if a similar compound contains the same core part and the same chiral atoms of carbapenem (Fig. 1b).

In order to estimate the noncovalent interactions, it is better to use noncovalent docking tools. The compounds obtained from the above design and similarity search were computed using two docking tools AutoDock Vina[29] and LeDock (http://www.lephar.com/)[30] with their default docking and scoring options. In order to avoid spatial blocking during molecular docking, the $-\mathrm{C} 7=\mathrm{O}$ part of ligand was removed (Fig. 1c). The details of the noncovalent dockings are described in Table S5.

For balancing the scores obtained from multiple docking and scoring systems, normalization and consensus scorings were usually employed.[31] We constructed two consensus scoring functions as follows:

$$
\begin{gathered}
\text { Score } 1=\left(E_{\text {Vina }}+E_{\text {LeDock }}\right) / 2 \\
\text { Score } 2=\frac{\bar{N}}{N} \text { Score } 1
\end{gathered}
$$

where $E_{\text {Vina }}$ is the normalized value of the score from AutoDock Vina, $E_{\text {LeDock }}$ the normalized value of the score from LeDock, $\mathrm{N}$ the number of the heavy atoms of a ligand and the average of all $\mathrm{N}$ numbers. Score1 is an average and Score2 is a normalized value with respect to the ligand size. Since the original scores from the docking outputs are negative, the normalization can be expressed as follows:

$$
E_{\text {norm }}=10 *\left(E-E_{\max }\right) /\left(E_{\max }-E_{\text {min }}\right)
$$

where $E$ is the score from the docking output for the considered ligand, $E_{\min }$ and $E_{\max }$ the minimum and the maximum of all scores for the calculated ligands, respectively. The factor 10 is used to tune the value to be close to the original scores. An ideal docking pose which should receive better scores simultaneously from the two consensus scoring functions, will be selected for further MD simulations and binding free energy calculations.

\section{Results and Discussion}

The flowchart in the present work is illustrated in Fig. 2. The basic process is that MD simulations and binding free energy calculations performed on the BlaC-carbapenem 
systems (Fig. 3) can provide a threshold for selecting hits. Then, the similarity search and combinations of structural units can be used to build new ligands which will be estimated using a series of computational treatments including molecular docking with consensus scoring and MD simulations with binding free energy calculations. The MD simulations were performed on the protein-ligand complexes with covalent bond which guarantees the stability of ligand in the binding site. The molecular dockings were performed on the noncovalent protein-ligand complexes, in which the covalent bond between protein and ligand was omitted and the atoms involved in the covalent bond were removed to avoid spatial repulsion during noncovalent docking. The noncovalent interactions are mainly evaluated using MD simulation and binding free energy, while the docking scores can be used as preliminary criteria for filtering a larger pool of molecular structures.

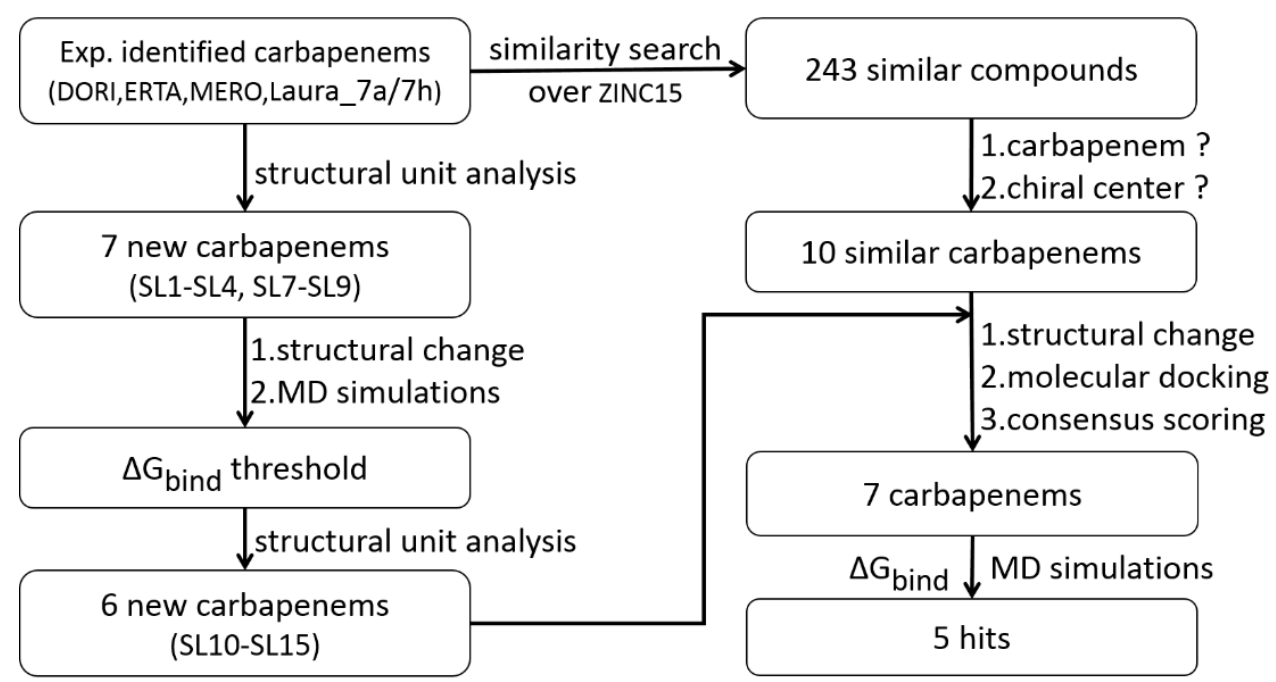

Fig. 2. Flowchart of the carbapenem design and evaluation approach.

\subsection{Carbapenems}

Based on the three known carbapenems, i.e. DORI, ERTA and MERO (Fig. 3a), four compounds, named SL1, SL2, SL3 and SL4 (Fig. 3b), have been built by combining the common parts of the R groups. SL1 contains only the core part of the carbapenem, without the $\mathrm{R}$ group. It will be used as a basis for comparison with those with different $\mathrm{R}$ groups. SL2 contains the pyrrolidin-3-ylthio moiety that is a common structural unit in the three $\mathrm{R}$ groups. SL3 is the common part of MERO and ERTA. SL4 contains the main part of DORI but removing the sulfamoxil-aminomethyl tail. Two more ligands Laura_7a and Laura_7h (Fig. 3c) were taken into consideration since they are also slow substrates with small $k_{\text {cat }}$ and $K_{\mathrm{m}}$, even smaller than those of the above three carbapenems.[12] The common part of Laura_7a and Laura_7h forms a new compound SL7 which contains the core part linked 
via a two-methylene chain to a triazole ring (Fig. 3d). SL8 and SL9 are formed from SL7 by deleting one- or two-methylene group, respectively (Fig. 3d). Another six compounds (SL10-SL15) (Fig. 3e) were built from the sub-structures of above twelve compounds.

(a)

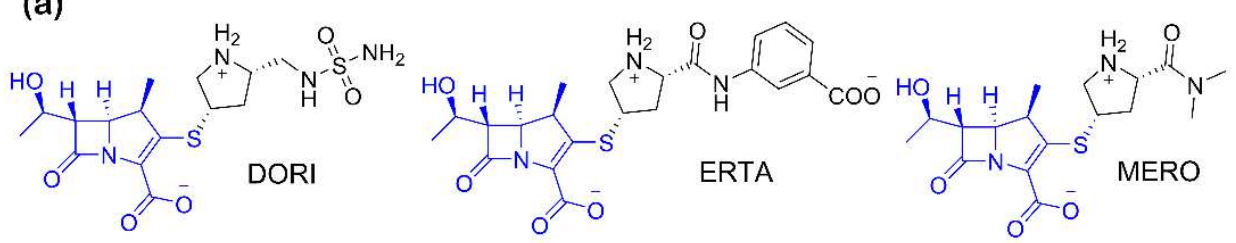

(b)
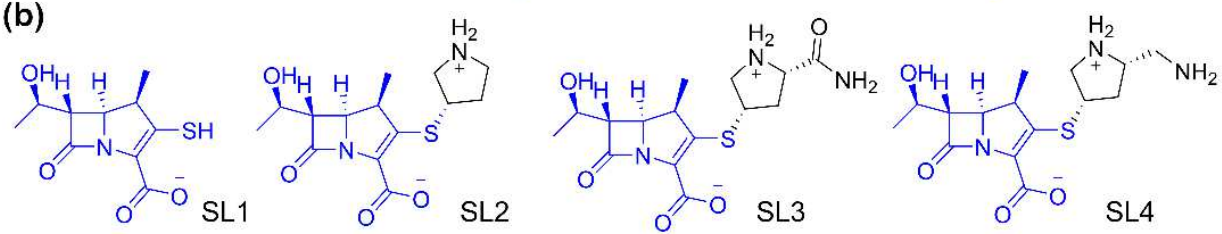

(c)

(d)
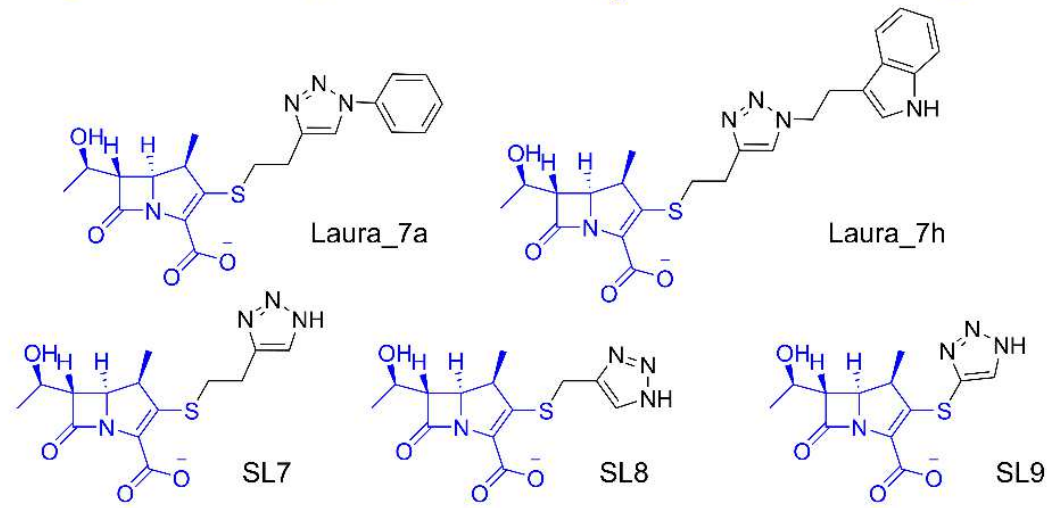

(e)
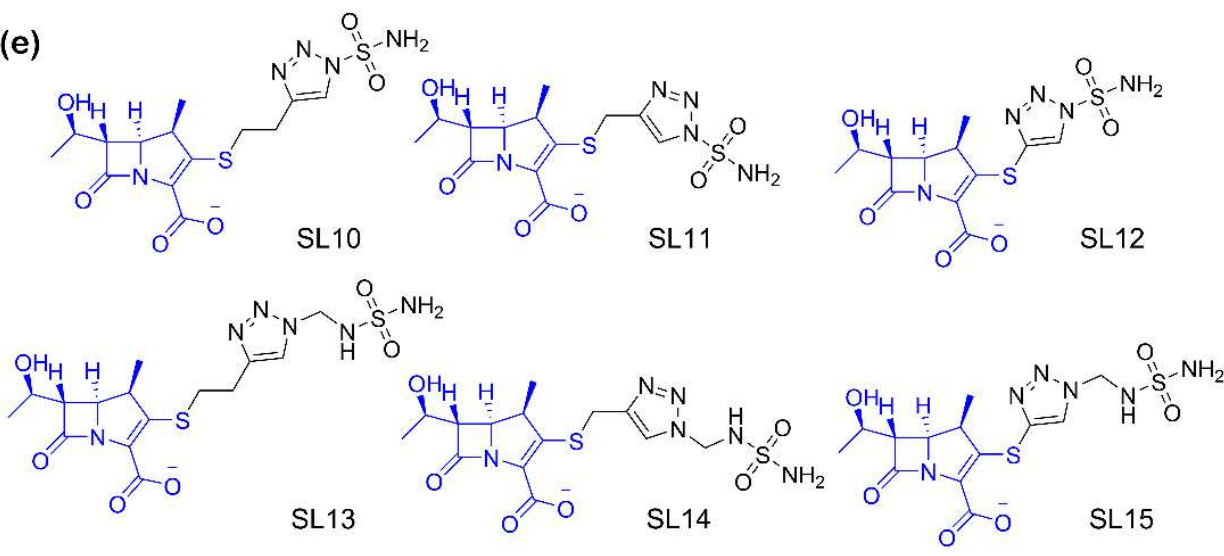

Fig. 3. Structures of carbapenems. 


\subsection{Binding free energy and slow substrates}

Three replicas of $50 \mathrm{~ns}$ MD simulations and the subsequent MM/GBSA binding free energy calculations were performed on each BlaC-ligand system. The averaged binding free energy derived from the three MD simulations will be used to evaluate the binding strength (Table 1). The standard deviations for the binding free energy are small, indicating the convergence of the binding free energy calculations (Table S6). More details of the binding free energies are shown in Table S7.

Initial analysis involved the five experimentally identified carbapenems including DORI, ERTA, MERO, Larua_7a, and Larua_7h (Fig. 3a and 3c), all with experimentally determined kinetic parameters.[12] A slow substrate has smaller $K_{\mathrm{m}}$ and smaller $k_{\text {cat. }}$ [12] It was indicated that a slow substrate exhibits slow tight-binding inhibition.[9,11] This correlates slow substrates with tight binding, i.e. more negative binding free energy. For the sake of comparison, it is convenient to list here the experimental kinetic parameters $\left(K_{\mathrm{m}}\right.$ in $\mu \mathrm{M}, k_{\mathrm{cat}} * 10^{3} \mathrm{in} \mathrm{s}^{-1}$ ) for binding and hydrolysis of the above carbapenems, i.e. four tight-binding slow substrates Laura_7h $(0.17,0.33)$, Laura_7a $(0.31,0.65)$, DORI $(0.46$, $0.69)$, MERO (0.92, 0.92), and one weaker binding substrate ERTA $(4.80,1.60)$. [12]

In Table 1, four tight-binding slow substrates have more negative binding free energy, i.e. DORI $-44.14 \mathrm{kcal} / \mathrm{mol}$, MERO $-36.44 \mathrm{kcal} / \mathrm{mol}$, Laura_7a $-36.70 \mathrm{kcal} / \mathrm{mol}$, and Laura_7h $-43.05 \mathrm{kcal} / \mathrm{mol}$, while the weaker binding substrate ERTA has a smaller binding free energy $-17.95 \mathrm{kcal} / \mathrm{mol}$. This indicates that a carbapenem analogs can be judged using the binding free energy. A small change in the structure of the $\mathrm{R}$ group might cause a large change in the binding free energy. The R groups of DORI, ERTA and MERO are similar. However, ERTA has a benzene ring with a carboxyl group and results at the less favorable binding free energy.

Table 1. Binding Free Energy Calculated from Three Replicas of $50 \mathrm{~ns}$ MD Simulations and the Average

\begin{tabular}{ccccc}
\hline \multirow{2}{*}{ Ligand } & \multicolumn{4}{c}{ Binding free energy (kcal/mol) } \\
\cline { 2 - 5 } & replica-1 & replica-2 & replica-3 & average \\
DORI & -43.66 & -45.38 & -43.38 & -44.14 \\
Laura_7h & -44.32 & -42.94 & -41.90 & -43.05 \\
SL7 & -34.17 & -40.78 & -38.54 & -37.83 \\
Laura_7a & -36.17 & -38.19 & -35.75 & -36.70 \\
MERO & -36.45 & -37.48 & -35.39 & -36.44 \\
SL1 & -36.02 & -35.56 & -37.21 & $-\mathbf{3 6 . 2 6}$ \\
SL3 & -36.35 & -36.18 & -31.11 & -34.55 \\
SL8 & -33.18 & -33.82 & -34.99 & -34.00 \\
SL9 & -33.22 & -32.80 & -35.71 & -33.91 \\
SL4 & -32.12 & -34.44 & -34.86 & -33.81 \\
SL2 & -33.61 & -31.60 & -29.19 & -31.47 \\
ERTA & -17.29 & -14.77 & -21.78 & -17.95 \\
\hline
\end{tabular}


It should be pointed out that the MD simulations of DORI and ERTA were performed on their own crystal structures, while all other carbapenems were placed on the crystal structure from the BlaC-MERO complex. Therefore, DORI and ERTA were submitted again for three replicas of $50 \mathrm{~ns}$ MD simulations using the protein structure from the BlaCMERO complex. The results are very close, i.e. $-43.83 \mathrm{kcal} / \mathrm{mol}$ for DORI vs. -44.14 $\mathrm{kcal} / \mathrm{mol}$ of Table 1 and $-15.11 \mathrm{kcal} / \mathrm{mol}$ for ERTA $v s$. $-17.95 \mathrm{kcal} / \mathrm{mol}$ of Table 1 . This also confirmed that the crystal structure from BlaC-MERO can be used as the representative for other carbapenems.

\subsection{New carbapenems}

\subsubsection{SL1-SL4 and the binding free energy threshold}

The structure of SL1 is just the core part of carbapenems (Fig. 3). The binding free energy of SL1 on $\mathrm{BlaC}$ is $-36.26 \mathrm{kcal} / \mathrm{mol}$ (Table 1 ), which can be used as a threshold to judge new carbapenems which contain different R groups (Fig. 3). An improved R group should receive a binding free energy more negative than this value. This value is already close to these values of the four good carbapenems. This means that the core part contributes the majority of binding free energy.

SL2, SL3 and SL4 (Fig. 3b) are constructed from the structural units of DORI and MERO. However, their binding free energy values have not been improved, -31.47 $\mathrm{kcal} / \mathrm{mol}$ for SL2, $-34.55 \mathrm{kcal} / \mathrm{mol}$ for SL3 and $-33.81 \mathrm{kcal} / \mathrm{mol}$ for SL4, respectively (Table 1). It is interesting that these values are even less than the energy of SL1. Comparing SL2SL4 with DORI, it can be seen that the sulfamoyl group $-\mathrm{NHSO}_{2} \mathrm{NH}_{2}$ or the sulfamoxilaminomethyl group $-\mathrm{CH}_{2} \mathrm{NHSO}_{2} \mathrm{NH}_{2}$ on DORI should be the important structural unit for contributing to the binding free energy.

\subsubsection{SL7-SL9}

By removing the benzene ring and indole ring from the R groups of Laura_7a and Laura_7h (Fig. 3c), we constructed SL7-SL9 (Fig. 3d), in which the triazole group is kept. There is an improvement in SL7 which has a binding free energy of $-37.83 \mathrm{kcal} / \mathrm{mol}$ (Table 1), more negative than that of SL1 $(-36.26 \mathrm{kcal} / \mathrm{mol})$. Further, we reduced the size of R groups for SL8 and SL9 since a smaller group retains more space for further optimization which will usually increase the ligand size, and present them here although the binding free energy values are temporarily not good for SL8 (-34.00 kcal/mol) and SL9 $(-33.91 \mathrm{kcal} / \mathrm{mol})$. Comparing the three structures of similar size, i.e. SL8, SL9 and SL2, the energy values of SL8 and SL9 are better than that of SL2. It can be seen that the triazole group $-\mathrm{C}_{2} \mathrm{H}_{2} \mathrm{~N}_{3}$ on SL8 and SL9 should be an important structural unit, while the pyrrolidine group $-\mathrm{C}_{4} \mathrm{H}_{9} \mathrm{~N}^{+}$ 
on SL2 is not so favorable. This is definitely proven by Laura_7a and Laura_7h which both have the triazole group and favorable binding free energy.

\subsubsection{SL10-SL15}

On the basis of above structural analyses, we picked up two important structural units as building blocks for constructing new carbapenems. One structural unit is the sulfamoyl group or its extended unit sulfamoxil-aminomethyl group on DORI. Another is the triazole group on Laura_7a and Laura_7h. Considering three different lengths of the linker from the core part, we can construct six new structures SL10-SL15 (Fig. 3e) via the combination of the triazole group and the sulfamoyl group or the sulfamoxil-aminomethyl group. The six carbapenem molecules will be evaluated later together with other compounds obtained from similarity search.

\subsection{Molecular docking}

The molecular dockings were performed on the above 6 new carbapenems SL10-SL15 (Fig. 3e) and 10 similar compounds Z1-Z10 (Fig. 4) which were obtained from the similarity search and visual inspection using the process described above. The names Z1Z10 are the abbreviations of their ZINC IDs (Table S8). As references, the five experimental carbapenems were also docked, including DORI, ERTA, MERO, Laura_7a and Laura_7h. For each ligand, multiple top binding poses from each docking tool were extracted for further selection. One filter is the RMSD of the core part with respect to the X-ray structure of MERO (PDB code: 3DWZ), which should be smaller than $2 \AA$ A Other filters are the consensus scores Score1 and Score2 which should be close to or even more negative than those of the identified MERO, i.e. Score $1<-4.93 \mathrm{kcal} / \mathrm{mol}$ and Score $2<-$ $4.78 \mathrm{kcal} / \mathrm{mol}$. Seven compounds meet the requirements, including SL10, SL11, SL12, SL13, SL14, SL15 and Z1 (Table 2), which will be further evaluated using MD simulations and MM/GBSA binding free energy calculations. 

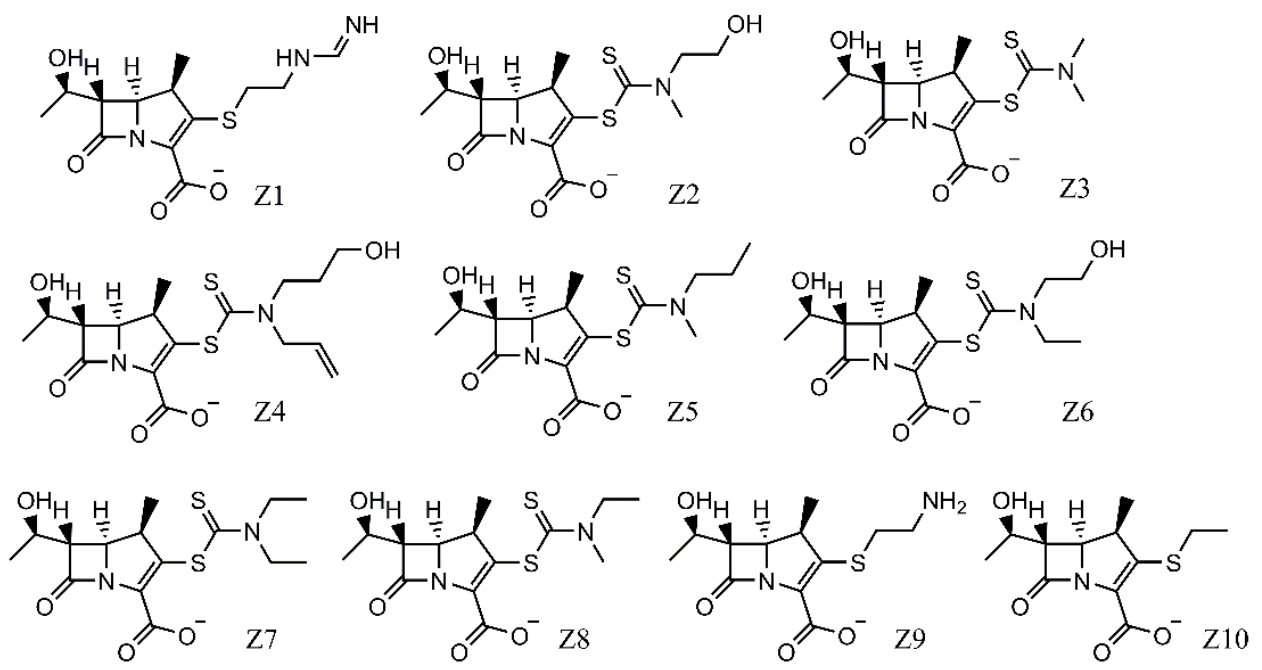

Fig. 4. Structures of the ten compounds obtained via similarity search over ZINC15 database.

Table 2. Scores of Molecular Dockings (kcal/mol)

\begin{tabular}{|c|c|c|c|c|c|c|c|}
\hline \multirow{2}{*}{ Ligand } & \multicolumn{2}{|c|}{ Vina score } & \multicolumn{2}{|c|}{ LeDock score } & \multirow{2}{*}{$\begin{array}{l}\text { heavy- } \\
\text { atoms }\end{array}$} & \multirow{2}{*}{ Score1 } & \multirow{2}{*}{ Score2 } \\
\hline & output & normalized & output & normalized & & & \\
\hline DORI & -7.3 & -7.6 & -7.47 & -8.52 & 25 & -8.06 & -7.50 \\
\hline ERTA & -7.9 & -10.0 & -6.09 & -4.42 & 31 & -7.21 & -5.41 \\
\hline MERO & -7.1 & -6.8 & -5.63 & -3.06 & 24 & -4.93 & -4.78 \\
\hline Laura_7a & -7.3 & -7.6 & $-^{a}$ & - & 27 & -7.60 & -6.55 \\
\hline Laura_7h & -7.7 & -9.2 & - & - & 32 & -9.20 & -6.69 \\
\hline SL10 & -7.2 & -7.2 & -7.21 & -7.74 & 25 & -7.47 & -6.96 \\
\hline SL11 & -7.2 & -7.2 & -7.19 & -7.69 & 24 & -7.44 & -7.22 \\
\hline SL12 & -7.5 & -8.4 & -7.13 & -7.51 & 23 & -7.95 & -8.05 \\
\hline SL13 & -7.4 & -8.0 & -7.76 & -9.38 & 27 & -8.69 & -7.49 \\
\hline SL14 & -7.3 & -7.6 & -7.52 & -8.66 & 26 & -8.13 & -7.28 \\
\hline SL15 & -7.8 & -9.6 & -7.97 & -10.00 & 25 & -9.80 & -9.12 \\
\hline $\mathrm{Z1}$ & -6.5 & -4.4 & -6.46 & -5.52 & 19 & -4.96 & -6.07 \\
\hline $\mathrm{Z} 2$ & -6.3 & -3.6 & -5.97 & -4.07 & 21 & -3.83 & -4.24 \\
\hline Z3 & -5.5 & -0.4 & -5.12 & -1.54 & 19 & -0.97 & -1.19 \\
\hline Z4 & -6.3 & -3.6 & -6.34 & -5.16 & 24 & -4.38 & -4.25 \\
\hline $\mathrm{Z5}$ & -6.3 & -3.6 & -5.52 & -2.73 & 21 & -3.16 & -3.50 \\
\hline Z6 & -6.4 & -4.0 & -5.91 & -3.89 & 22 & -3.94 & -4.17 \\
\hline $\mathrm{Z7}$ & -5.5 & -0.4 & -5.19 & -1.75 & 21 & -1.08 & -1.19 \\
\hline Z8 & -6.2 & -3.2 & -5.58 & -2.91 & 20 & -3.05 & -3.55 \\
\hline
\end{tabular}




\begin{tabular}{lrrrrrrr}
\hline Z9 & -6.0 & -2.4 & -5.94 & -3.98 & 17 & -3.19 & -4.36 \\
Z10 & -5.4 & 0.0 & -4.60 & 0.00 & 16 & 0.00 & 0.00 \\
\hline
\end{tabular}

Notes: ${ }^{a}$ Laura_7a and Laura_7h don't have the LeDock conformations with RMSD less than $2 \AA$.

\subsection{Binding free energy for S10-S15 and Z1}

The binding free energy values of the seven new compounds are shown in Table 3. Comparing with the SL1 threshold $-36.26 \mathrm{kcal} / \mathrm{mol}$ (Table 1), it is easy to pick up four tight-binding compounds, i.e. SL10 (-41.69 kcal/mol), SL12 (-39.47 kcal/mol), SL14 ($40.05 \mathrm{kcal} / \mathrm{mol})$ and SL15 $(-44.22 \mathrm{kcal} / \mathrm{mol})$. In addition, SL7 might provide a parent framework for further development since the binding free energy $-37.83 \mathrm{kcal} / \mathrm{mol}$ is also favorable (Table 1).

Table 3. Binding Free Energies (kcal/mol) of New Carbapenems Calculated from Three Replicas of $50 \mathrm{~ns}$ MD Simulations and the Average

\begin{tabular}{lllll}
\hline \multirow{2}{*}{ Ligand } & \multicolumn{4}{l}{ Binding free energy $(\mathrm{kcal} / \mathrm{mol})$} \\
\cline { 2 - 5 } & replica-1 & replica-2 & replica-3 & average \\
\hline SL10 & -42.40 & -41.21 & -41.47 & -41.69 \\
SL11 & -22.90 & -20.73 & -24.93 & -22.85 \\
SL12 & -39.25 & -39.50 & -39.66 & -39.47 \\
SL13 & -30.75 & -29.43 & -30.03 & -30.07 \\
SL14 & -40.23 & -38.51 & -41.40 & -40.05 \\
SL15 & -45.75 & -42.47 & -44.43 & -44.22 \\
Z1 & -31.80 & -33.35 & -36.19 & -33.78 \\
\hline
\end{tabular}

\subsection{Stability, Hydrogen Bond and Binding Pose}

Small RMSD values (Table S9) guarantee the stability of MD trajectory for binding free energy calculations. The RMSF values of ligands (Fig. S1 S3) indicate that the core structures are conserved in the binding site while the R groups are more flexible, associated with them being more exposed to the solvent.

During MD simulation, atoms are constantly moving within a small region and some inter-atomic distances keep changing. Thus, the number of H-bonds will constantly change. Therefore, we use the statistical number of $\mathrm{H}$-bonds which is the count of $\mathrm{H}$-bonds divided by the number of snapshots (i.e. 5,000). Table 4 provides the averaged numbers of $\mathrm{H}$-bonds over the three MD simulations, which details for the three MD replicas are given in Table S10. The H-bonds can be categorized into three types, i.e. core-protein, R-protein and ligand-water which mostly comes from the R-water part. A tight-binding carbapenem should have stable core-protein H-bonds, such as SL7, SL10, SL12 and SL15 which have about 5 core-protein H-bonds. A flexible carbapenem might have more $\mathrm{H}$-bonds with water 
which tends to pull ligand out of the binding site, thereby reducing the binding free energy, such as ERTA which has about $10 \mathrm{H}$-bonds with water. Fig. S4 and S5 shows the H-bonds and hydrophobic interactions for SL15 and ERTA, respectively, based on their representative structures which are selected via clustering the conformations extracted from the MD trajectories.

Table 4. Averaged Numbers of H-Bonds between Protein BlaC and Ligand Carbapenems over 5,000 Snapshots Extracted from Each of the Three Replicas of $50 \mathrm{~ns}$ MD Simulations

\begin{tabular}{llll}
\hline \multirow{2}{*}{ Ligand } & \multicolumn{2}{l}{ No. H-bonds in ligand-BlaC } & \multirow{2}{*}{$\begin{array}{l}\text { No. H-bonds in ligand- } \\
\text { water }\end{array}$} \\
\cline { 2 - 3 } & core-BlaC & R-BlaC & 3.94 \\
\hline DORE & 3.93 & 0.59 & 9.89 \\
ERTA & 2.91 & 0.32 & 2.71 \\
MERO & 4.08 & 0.04 & 1.16 \\
SL1 & 4.44 & 0.00 & 2.10 \\
SL2 & 4.32 & 0.02 & 2.08 \\
SL3 & 4.48 & 1.37 & 3.57 \\
SL4 & 4.13 & 0.74 & 2.58 \\
Laura_7a & 4.28 & 0.02 & 3.21 \\
Laura_7h & 4.26 & 0.06 & 3.03 \\
SL7 & 4.41 & 0.33 & 2.57 \\
SL8 & 4.70 & 0.18 & 2.93 \\
SL9 & 4.58 & 0.02 & 3.92 \\
SL10 & 4.37 & 0.61 & 5.17 \\
SL11 & 3.16 & 0.23 & 3.51 \\
SL12 & 4.69 & 0.69 & 7.05 \\
SL13 & 2.94 & 0.60 & 5.28 \\
SL14 & 3.77 & 1.18 & 3.75 \\
SL15 & 4.73 & 0.78 & 2.76 \\
Z1 & 4.60 & 0.19 & \\
\hline & & & \\
\hline
\end{tabular}

The binding poses of five selected carbapenems, SL7, SL10, SL12, SL14 and SL15, are expressed in Fig. 5 using the representative conformations which are superimposed with respect to the binding site residues. The locations of the ligands are marked using three residues, Ser84 in red, Thr231 in dodger blue and Glu292 in spring green, respectively. All core parts are stably laying at the central binding site near Ser84. The R groups however spread toward two different directions, SL14 toward Thr231 and other four toward Glu292. 


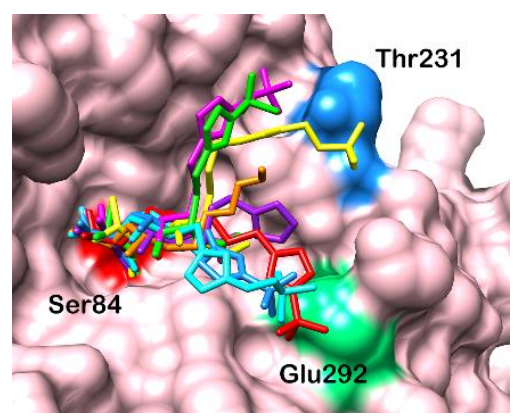

Fig. 5. Binding poses of five new compounds, SL7, SL10, SL12, SL14 and SL15. The figure was created using Chimera[32].

\section{Conclusions}

This research provides a feasible approach to design covalent drug, based on two issues, 1) a hypothesis that the noncovalent interactions can be used to compare the binding strength if the covalent bonding is same to all ligands, 2) a computational protocol combining MD simulations, binding free energy calculations, similarity search, molecular docking and consensus scoring. MD simulations and MM/GBSA binding free energy calculations were first applied to the experimentally identified carbapenems to obtain a threshold for selecting novel carbapenem ligands. The structural units extracted from the experimental carbapenems were used to construct new carbapenem compounds. These structural units contain triazole ring, sulfamoxil-aminomethyl and sulfamoyl groups. In addition, more compounds have been obtained from the similarity search over the ZINC15 database. All the new compounds have been evaluated using AutoDock Vina and LeDock dockings and our consensus scores, followed by MD simulations and binding free energy calculations. Finally, five new compounds SL7, SL10, SL12, SL14, and SL15 were suggested for further experimental validations.

It was expected to find some compounds, but not any herein, from the similarity search over the ZINC database which contains more than ten million chemical compounds. It seems that our method will be helpful to design totally novel compounds that are not in a database. Of course, a huge database such as ZINC should be suitable for virtual screening of other scaffolds for designing non-lactam BlaC inhibitors which can be an alternate way to improve TB treatment.[33-36] Some $\beta$-lactam BlaC inhibitors were not computed in this work since they don't possess the same core structure.[37]

The covalent bond is built between the serine hydroxyl and the lactam carbonyl using a new residue SEP with force filed parameters only suitable for these specific carbapenem structures. Opportunely, these parameters gave proper binding free energy values in agreement with the experimental data and were successfully applied to the MD simulations of these carbapenems. For a wide range of applications, these parameters need to be tested for more observable quantities using sophisticated protocols such as those steps used in the 
development of CGenFF[38] and its extension DGenFF which is developed using a polarizable force field based on classical Drude oscillator model.[39]

\section{Supporting Information.}

The Supporting Information is available free of charge:

\section{Corresponding Author}

*E-mail: sjzhong@dlut.edu.cn

\section{ORCID}

Shijun Zhong: 0000-0002-4213-2808

\section{ResearcherID}

Shijun Zhong: P-5280-2018

\section{Present Address}

Shijun Zhong: School of Bioengineering, Dalian University of Technology, Dalian, Liaoning, 116024, P. R. China. Email: sjzhong@dlut.edu.cn, phone: +86 13654922395, fax: +86 411-84708083.

Lu Sun: School of Bioengineering, Dalian University of Technology, Dalian, Liaoning, 116024, P. R. China. Email: sunlu@ mail.dlut.edu.cn, phone: +86 15941129376, fax: +86 411-84708083. 
Hongjun Fan: The State Key Lab of Molecular Reaction Dynamics, Dalian Institute of

Chemical Physics (iCheM), Chinese Academy of Sciences, Dalian, Liaoning, 116023, P.

R. China. Email: fanhj@ dicp.ac.cn, phone: +86 13840937024, fax: +86 411- 84691570.

\section{Author Contributions}

Sun performed the computations and analyzed the data. Sun, Fan and Zhong designed the research protocol. Sun and Zhong wrote the paper.

\section{Funding Sources}

This work was supported by the Fundamental Research Funds for the Central Universities (grant DUT19LAB21 to SZ).

\section{Notes}

The authors declare no competing financial interest.

\section{Acknowledgments}

This work was supported by the Fundamental Research Funds for the Central Universities (grant DUT19LAB21 to SZ) and the Supercomputing Center of Dalian University of Technology. We thank Alexander D. MacKerell, Jr. for helpful discussions.

\section{Abbreviations}

BlaC, Class A $\beta$-lactamase; DORI, doripenem; ERTA, ertapenem; ESP, molecular electrostatic potential; MERO, meropenem; MD, molecular dynamics; MM/GBSA, Molecular Mechanics/Generalized Born Surface Area; PDB, Protein Data Bank; RESP, restrained electrostatic potential; RMSD, root mean square deviation; RMSF, root mean square fluctuation.

\section{References}


1. World-Health-Organization (2018) Global tuberculosis report 2018. Global tuberculosis report.

2. Chiang CY, Centis R, Migliori GB (2010) Drug-resistant tuberculosis: Past, present, future. Respirology 15 (3):413-432

3. Ealand CS, Machowski EE, Kana BD (2018) beta-lactam resistance: The role of low molecular weight penicillin binding proteins, beta-lactamases and ld-transpeptidases in bacteria associated with respiratory tract infections. IUBMB life 70 (9):855-868. doi:10.1002/iub.1761

4. Cho H, Uehara T, Bernhardt TG (2014) Beta-lactam antibiotics induce a lethal malfunctioning of the bacterial cell wall synthesis machinery. Cell 159 (6):1300-1311. doi:10.1016/j.cell.2014.11.017

5. Fisher JF, Meroueh SO, Mobashery S (2005) Bacterial resistance to beta-lactam antibiotics: Compelling opportunism, compelling opportunity. Chem Rev 105 (2):395-424

6. Meroueh SO, Fisher JF, Schlegel HB, Mobashery S (2005) Ab initio QM/MM study of class A beta-lactamase acylation: Dual participation of Glu166 and Lys73 in a concerted base promotion of Ser70. J Am Chem Soc 127 (44):15397-15407

7. Hermann JC, Ridder L, Hotje HD, Mulholland AJ (2006) Molecular mechanisms of antibiotic resistance: QM/MM modelling of deacylation in a class A beta-lactamase. Org Biomol Chem 4 (2):206-210

8. Tehrani K, Martin NI (2018) beta-lactam/beta-lactamase inhibitor combinations: an update. MedChemComm 9 (9):1439-1456. doi:10.1039/c8md00342d

9. Hugonnet JE, Tremblay LW, Boshoff HI, Barry CE, Blanchard JS (2009) Meropenem-Clavulanate Is Effective Against Extensively Drug-Resistant Mycobacterium tuberculosis. Science 323 (5918):1215-1218

10. El-Gamal MI, Brahim I, Hisham N, Aladdin R, Mohammed H, Bahaaeldin A (2017) Recent updates of carbapenem antibiotics. Eur J Med Chem 131:185-195

11. Tremblay LW, Fan F, Blanchard JS (2010) Biochemical and Structural Characterization of Mycobacterium tuberculosis beta-Lactamase with the Carbapenems Ertapenem and Doripenem. Biochemistry-Us 49 (17):3766-3773

12. Iannazzo L, Soroka D, Triboulet S, Fonvielle M, Compain F, Dubee V, Mainardi JL, Hugonnet JE, Braud E, Arthur M, Etheve-Quelquejeu M (2016) Routes of Synthesis of Carbapenems for Optimizing Both the Inactivation of L,D-Transpeptidase Ldt(Mt1) of Mycobacterium tuberculosis and the Stability toward Hydrolysis by beta-Lactamase BlaC. J Med Chem 59 (7):3427-3438

13. Ambler RP (1980) The structure of beta-lactamases. Philosophical transactions of the Royal Society of London Series B, Biological sciences 289 (1036):321-331. doi:10.1098/rstb.1980.0049

14. Ouyang XC, Zhou S, Su CTT, Ge ZM, Li RT, Kwoh CK (2013) CovalentDock: Automated covalent docking with parameterized covalent linkage energy estimation and molecular geometry constraints. J Comput Chem 34 (4):326-336

15. Zhu K, Bonelli KW, Greenwood JR, Day T, Abel R, Farid RS, Harder E (2014) Docking Covalent Inhibitors: A Parameter Free Approach To Pose Prediction and Scoring. J Chem Inf Model 54 (7):1932-1940

16. Chatterjee P, Botello-Smith WM, Zhang H, Qian L, Alsamarah A, Kent D, Lacroix JJ, Baudry M, Luo Y (2017) Can Relative Binding Free Energy Predict Selectivity of Reversible Covalent Inhibitors? J Am Chem Soc 139 (49):17945-17952. doi:10.1021/jacs.7b08938

17. Sterling T, Irwin JJ (2015) ZINC 15-Ligand Discovery for Everyone. J Chem Inf Model 55 (11):2324-2337

18. Berman HM, Westbrook J, Feng Z, Gilliland G, Bhat TN, Weissig H, Shindyalov IN, Bourne PE (2000) The Protein Data Bank. Nucleic Acids Res 28 (1):235-242 
19. D.A. Case VB, J.T. Berryman, R.M. Betz, Q. Cai, D.S. Cerutti, T.E. Cheatham, III, T.A. Darden, R.E. Duke, H. Gohlke, A.W. Goetz, S. Gusarov, N. Homeyer, P. Janowski, J. Kaus, I. Kolossváry, A. Kovalenko, T.S. Lee, S. LeGrand, T. Luchko, R. Luo, B. Madej, K.M. Merz, F. Paesani, D.R. Roe, A. Roitberg, C. Sagui, R. Salomon-Ferrer, G. Seabra, C.L. Simmerling, W. Smith, J. Swails, R.C. Walker, J. Wang, R.M. Wolf, X. Wu and P.A. Kollman (2014), AMBER 14, University of California, San Francisco.

20. Baker EN, Hubbard RE (1984) Hydrogen-Bonding in Globular-Proteins. Prog Biophys Mol Bio 44 (2):97-179. doi:Doi 10.1016/0079-6107(84)90007-5

21. M. J. Frisch GWT, H. B. Schlegel, G. E. Scuseria, M. A. Robb, J. R. Cheeseman, G. Scalmani, V. Barone, B. Mennucci, G. A. Petersson, H. Nakatsuji, M. Caricato, X. Li, H. P. Hratchian, A. F. Izmaylov, J. Bloino, G. Zheng, J. L. Sonnenberg, M. Hada, M. Ehara, K. Toyota, R. Fukuda, J. Hasegawa, M. Ishida, T. Nakajima, Y. Honda, O. Kitao, H. Nakai, T. Vreven, J. A. Montgomery, Jr., J. E. Peralta, F. Ogliaro, M. Bearpark, J. J. Heyd, E. Brothers, K. N. Kudin, V. N. Staroverov, T. Keith, R. Kobayashi, J. Normand, K. Raghavachari, A. Rendell, J. C. Burant, S. S. Iyengar, J. Tomasi, M. Cossi, N. Rega, J. M. Millam, M. Klene, J. E. Knox, J. B. Cross, V. Bakken, C. Adamo, J. Jaramillo, R. Gomperts, R. E. Stratmann, O. Yazyev, A. J. Austin, R. Cammi, C. Pomelli, J. W. Ochterski, R. L. Martin, K. Morokuma, V. G. Zakrzewski, G. A. Voth, P. Salvador, J. J. Dannenberg, S. Dapprich, A. D. Daniels, Ö. Farkas, J. B. Foresman, J. V. Ortiz, J. Cioslowski and D. J. Fox, Gaussian 09, Revision C.01, Gaussian, Inc., Wallingford CT, 2009.

22. Hornak V, Abel R, Okur A, Strockbine B, Roitberg A, Simmerling C (2006) Comparison of multiple amber force fields and development of improved protein backbone parameters. Proteins 65 (3):712-725

23. Wang JM, Wolf RM, Caldwell JW, Kollman PA, Case DA (2004) Development and testing of a general amber force field. J Comput Chem 25 (9):1157-1174

24. Roe DR, Cheatham TE (2013) PTRAJ and CPPTRAJ: Software for Processing and Analysis of Molecular Dynamics Trajectory Data. J Chem Theory Comput 9 (7):3084-3095

25. Kelley LA, Gardner SP, Sutcliffe MJ (1996) An automated approach for clustering an ensemble of NMR-derived protein structures into conformationally related subfamilies. Protein Eng 9 (11):1063-1065

26. Kollman PA, Massova I, Reyes C, Kuhn B, Huo SH, Chong L, Lee M, Lee T, Duan Y, Wang W, Donini O, Cieplak P, Srinivasan J, Case DA, Cheatham TE (2000) Calculating structures and free energies of complex molecules: Combining molecular mechanics and continuum models. Accounts Chem Res 33 (12):889-897

27. Onufriev A, Bashford D, Case DA (2004) Exploring protein native states and large-scale conformational changes with a modified generalized born model. Proteins 55 (2):383-394

28. Weiser J, Shenkin PS, Still WC (1999) Approximate atomic surfaces from linear combinations of pairwise overlaps (LCPO). J Comput Chem 20 (2):217-230

29. Trott O, Olson AJ (2010) Software News and Update AutoDock Vina: Improving the Speed and Accuracy of Docking with a New Scoring Function, Efficient Optimization, and Multithreading. J Comput Chem 31 (2):455-461

30. Zhao HT, Caflisch A (2013) Discovery of ZAP70 inhibitors by high-throughput docking into a conformation of its kinase domain generated by molecular dynamics. Bioorg Med Chem Lett 23 (20):5721-5726

31. Zhong S, Zhang Y, Xiu Z (2010) Rescoring ligand docking poses. Current opinion in drug discovery \& development 13 (3):326-334 
32. Pettersen EF, Goddard TD, Huang CC, Couch GS, Greenblatt DM, Meng EC, Ferrin TE (2004) UCSF chimera - A visualization system for exploratory research and analysis. J Comput Chem 25 (13):1605-1612

33. Sagar A, Haleem N, Bashir YM, Ashish (2017) Search for non-lactam inhibitors of mtb betalactamase led to its open shape in apo state: new concept for antibiotic design. Scientific reports 7 (1):6204. doi:10.1038/s41598-017-06023-3

34. Soroka D, Ourghanlian C, Compain F, Fichini M, Dubee V, Mainardi JL, Hugonnet JE, Arthur M (2017) Inhibition of beta-lactamases of mycobacteria by avibactam and clavulanate. The Journal of antimicrobial chemotherapy 72 (4):1081-1088. doi:10.1093/jac/dkw546

35. Hazra S, Kurz SG, Wolff K, Nguyen L, Bonomo RA, Blanchard JS (2015) Kinetic and Structural Characterization of the Interaction of 6-Methylidene Penem 2 with the beta-Lactamase from Mycobacterium tuberculosis. Biochemistry-Us 54 (36):5657-5664

36. Kurz SG, Hazra S, Bethel CR, Romagnoli C, Caselli E, Prati F, Blanchard JS, Bonomo RA (2015) Inhibiting the beta-Lactamase of Mycobacterium tuberculosis (Mtb) with Novel Boronic Acid Transition-State Inhibitors (BATSIs). Acs Infect Dis 1 (6):234-242

37. Tassoni R, Blok A, Pannu NS, Ubbink M (2019) New Conformations of Acylation Adducts of Inhibitors of beta-Lactamase from Mycobacterium tuberculosis. Biochemistry-Us 58 (7):997-1009. doi:10.1021/acs.biochem.8b01085

38. Vanommeslaeghe K, Hatcher E, Acharya C, Kundu S, Zhong S, Shim J, Darian E, Guvench O, Lopes P, Vorobyov I, Mackerell AD, Jr. (2010) CHARMM general force field: A force field for drug-like molecules compatible with the CHARMM all-atom additive biological force fields. $\mathrm{J}$ Comput Chem 31 (4):671-690. doi:10.1002/jcc.21367

39. Lemkul JA, Huang J, Roux B, MacKerell AD, Jr. (2016) An Empirical Polarizable Force Field Based on the Classical Drude Oscillator Model: Development History and Recent Applications. Chem Rev 116 (9):4983-5013. doi:10.1021/acs.chemrev.5b00505 


\section{Figures}
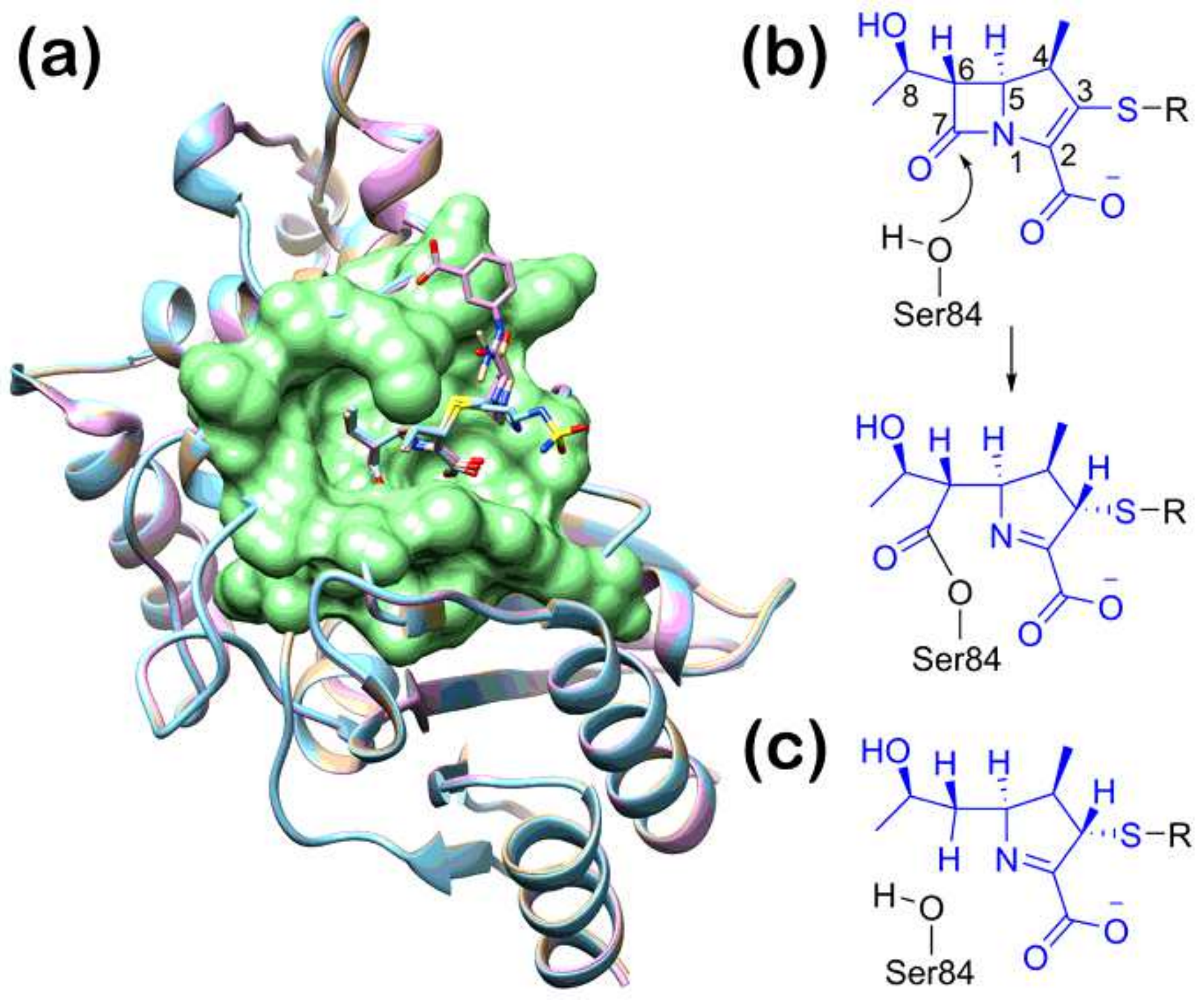

Figure 1

(a) Superimposition of the three BlaC-carbapenem crystal structures. BlaC is shown in cartoon while the carbapenems are shown as sticks (DORI carbons in sky blue, ERTA carbons in plum and MERO carbons in tan). The binding site is expressed in surface using the structure of BlaC-MERO (PDB ID: 3DWZ). (b) Structural change during the covalent binding, i.e. the opening of $\beta$-lactam ring and the isomerization of pyrroline ring. (c) Structure used in molecular dockings.

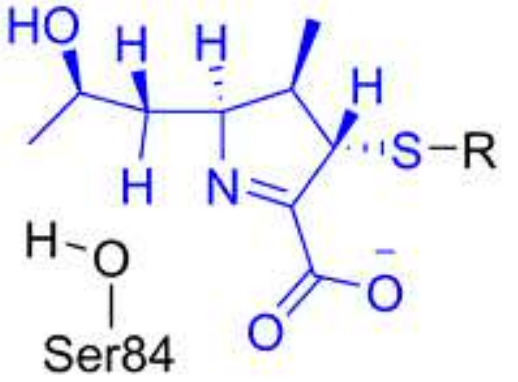




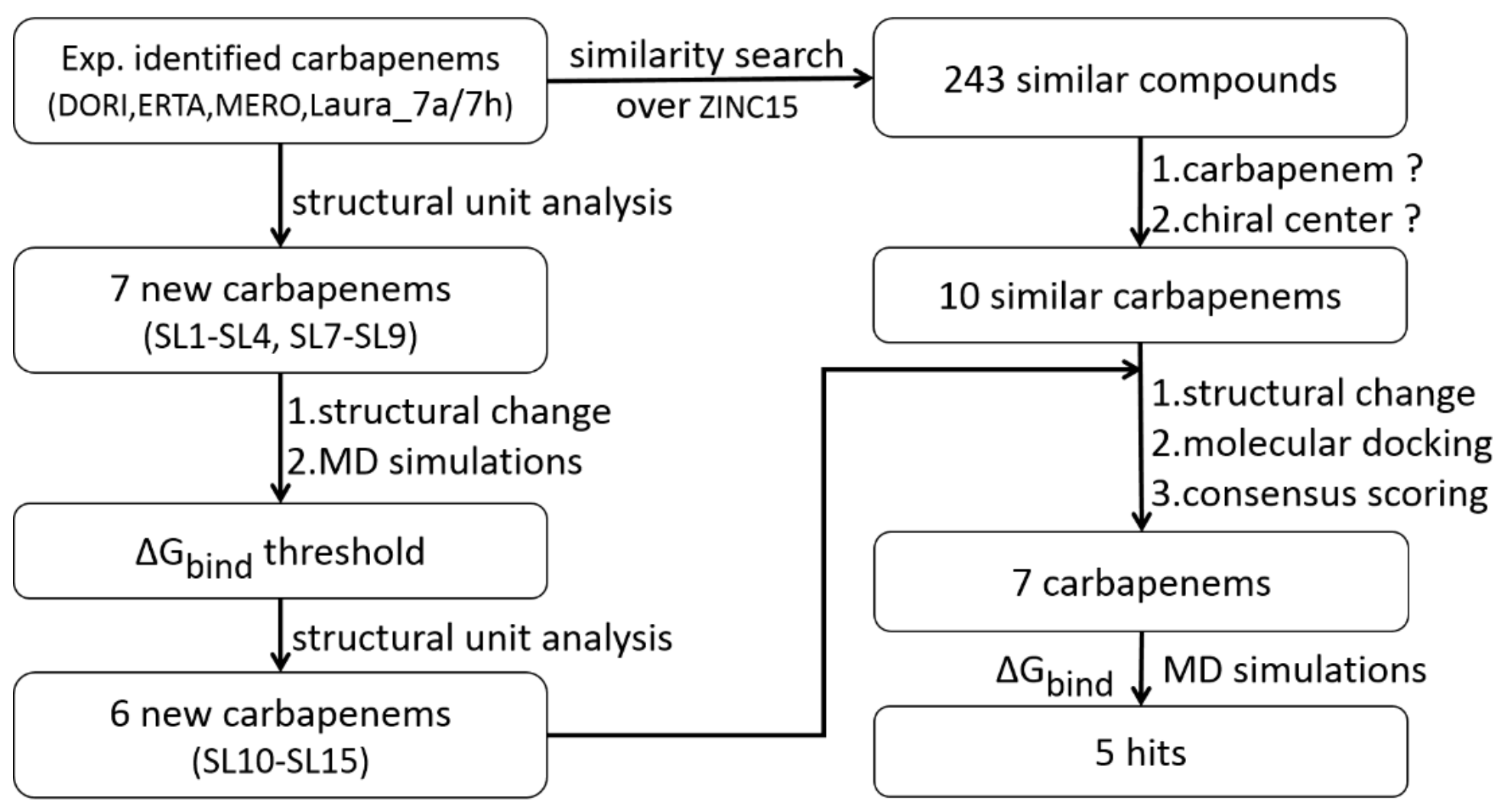

Figure 2

Flowchart of the carbapenem design and evaluation approach. 
(a)

(n)

(b)
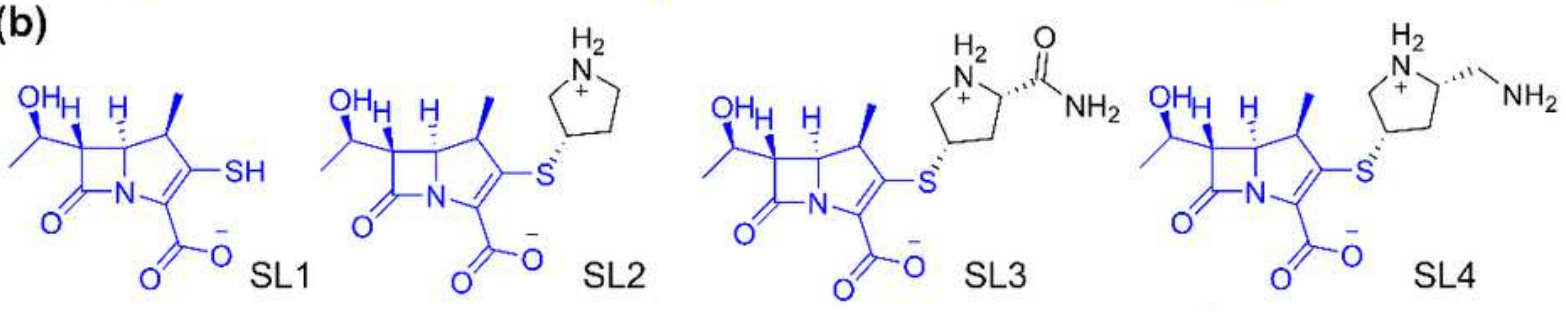

(c)

(d)
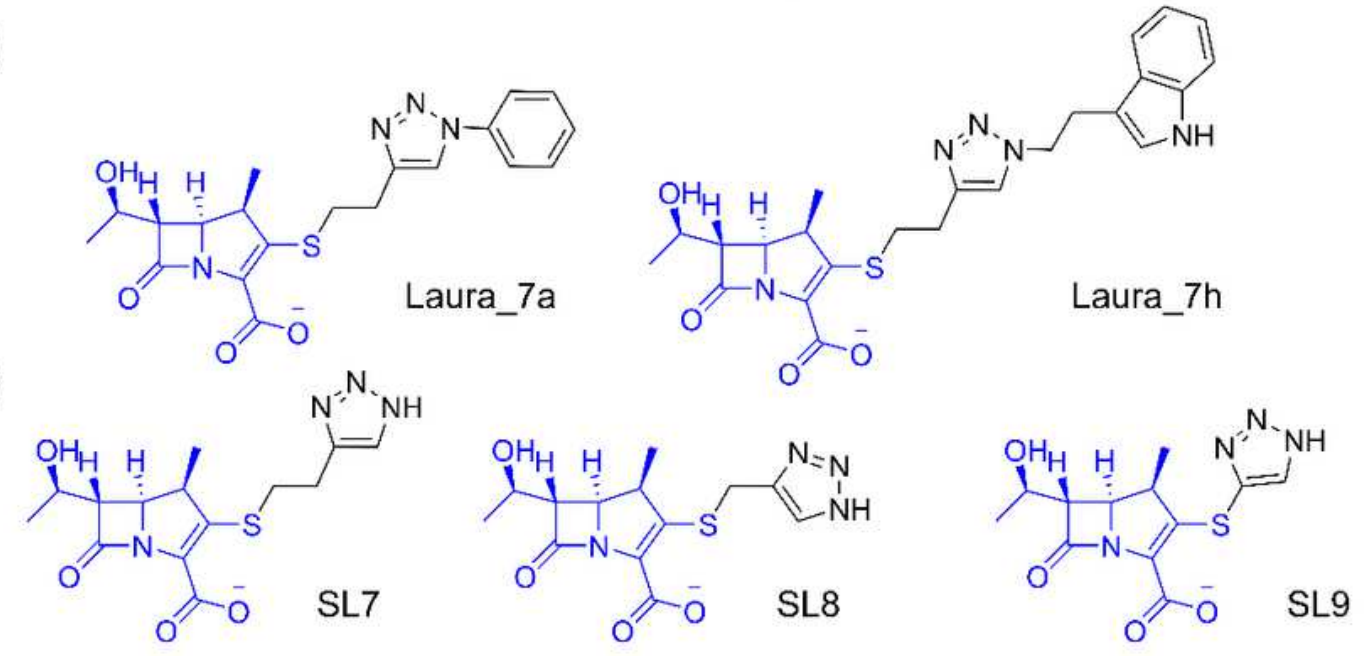

(e)
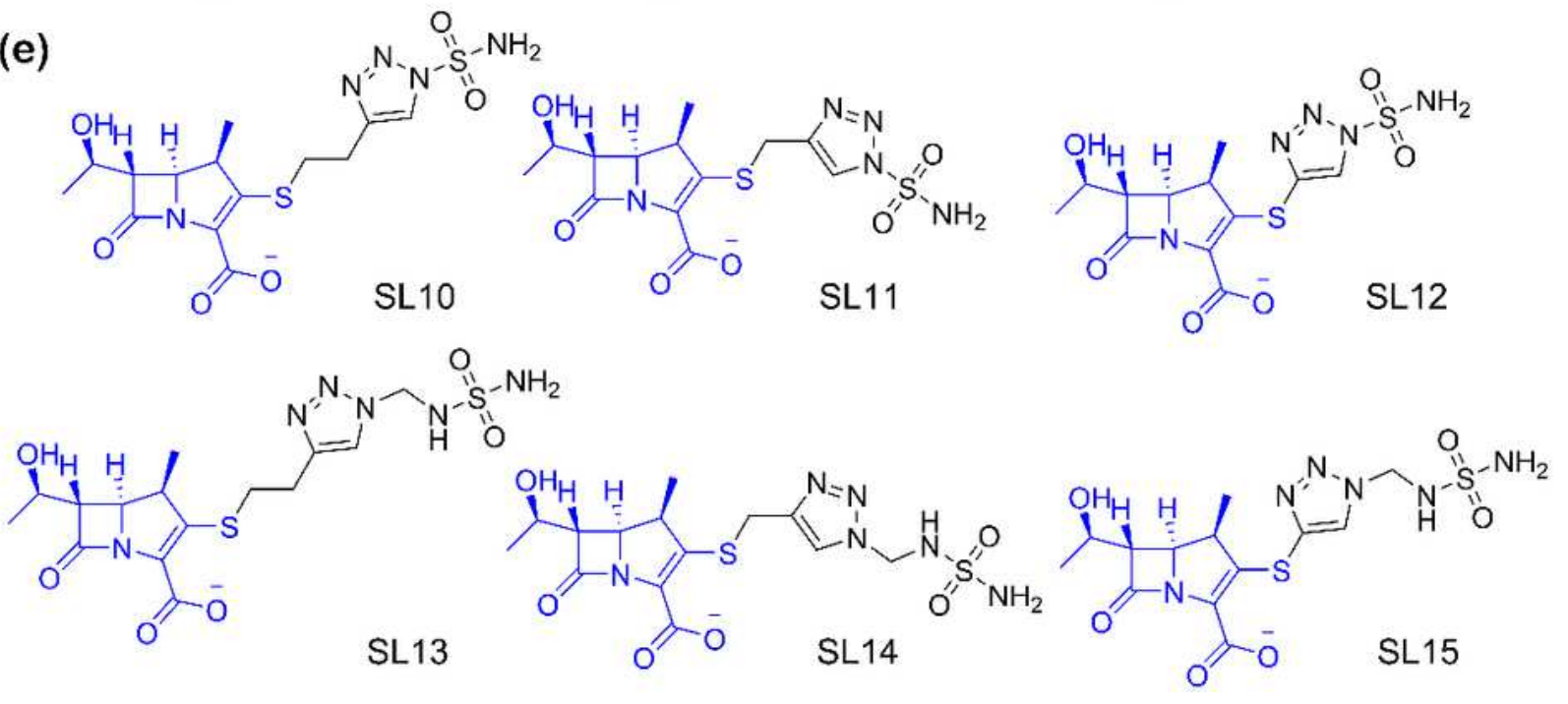

Figure 3

Structures of carbapenems. 

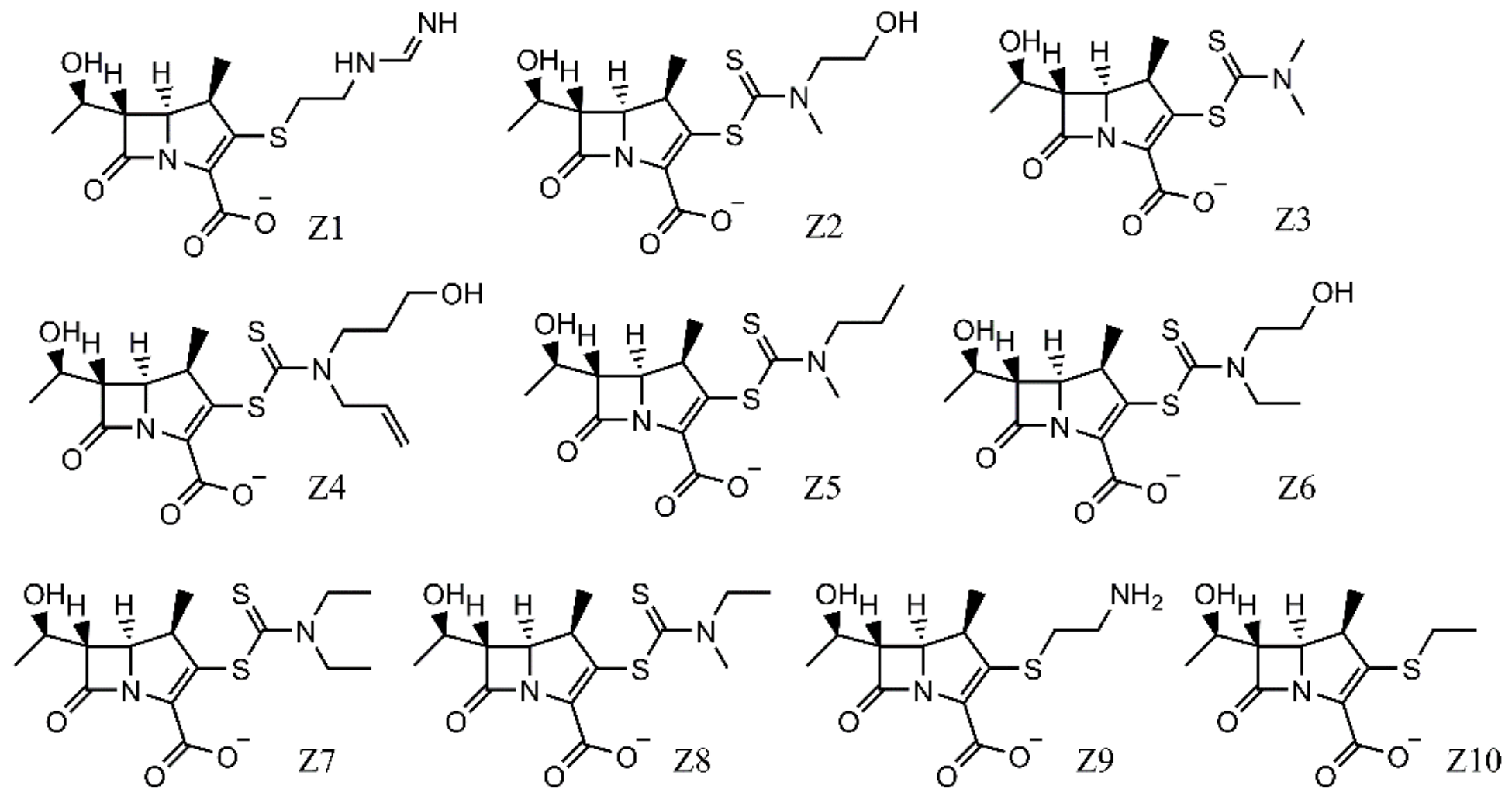

Figure 4

Structures of the ten compounds obtained via similarity search over ZINC15 database.

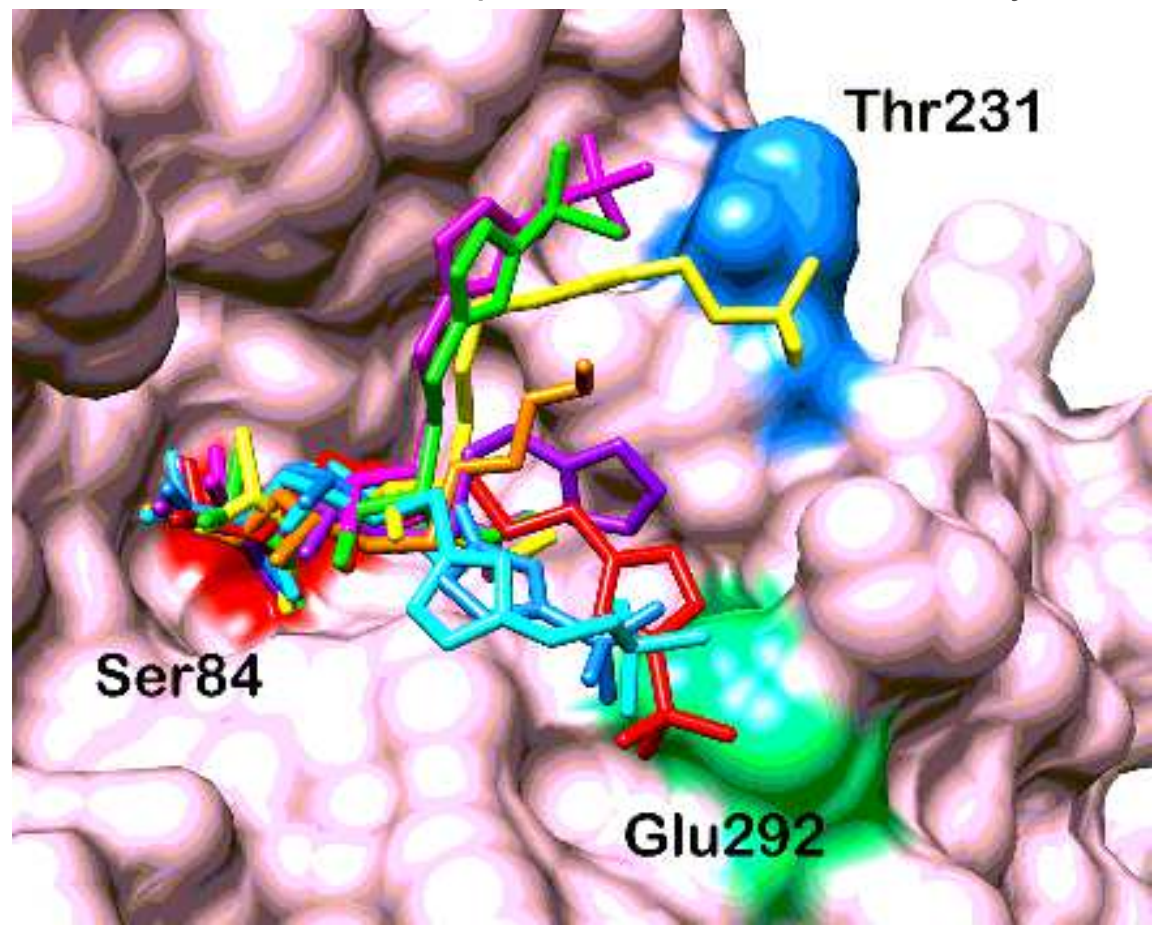

Figure 5

Binding poses of five new compounds, SL7, SL10, SL12, SL14 and SL15. The figure was created using Chimera[32]. 


\section{Supplementary Files}

This is a list of supplementary files associated with this preprint. Click to download.

- jmmCarbapenemSupportingInformation.docx 